\title{
Comparing signers and speakers: Building a directly comparable corpus of Auslan and Australian English ${ }^{1}$
}

Gabrielle Hodge, La Trobe University, Melbourne

Kazuki Sekine, Radboud University, Max Planck Institute for Psycholinguistics, Nijmegen

Adam Schembri, University of Birmingham, United Kingdom

Trevor Johnston, Macquarie University, Sydney

Pre-publication draft of accepted version, to appear in Corpora (14)1

\begin{abstract}
The Auslan and Australian English archive and corpus is the first bilingual, multimodal documentation of a deaf signed language (Auslan, the language of the Australian deaf community) and its ambient spoken language (Australian English). It aims to facilitate the direct comparison of face-to-face, multimodal talk produced by deaf signers and hearing speakers from the same city. Here we describe the documentation of the bilingual, multimodal archive and outline its development pathway into a directly comparable corpus of a signed language and spoken language. We differentiate it from existing bilingual corpora and offer some research questions for which the resulting corpus may be best placed to answer. The Auslan and Australian English corpus has the potential to redress several significant misunderstandings in the comparison of signed and spoken languages, especially those that follow from misapplications of the paradigm that multimodal signed languages are used and structured in ways that are parallel to the unimodal spoken or written conventions of spoken languages.
\end{abstract}

Key words: comparable corpora, multimodal, sign language, spoken language, deaf

\section{Introduction}

What are the similarities and differences between signed languages (SLs) and spoken languages (SpLs)? Do similarities outweigh perceived differences, suggesting few (if any) modality effects in the overall documentation of language diversity and variation? Or does SL structure in fact differ significantly from SpL structure? These questions (among others) have long fascinated researchers interested in the SLs of deaf communities (e.g. Bellugi \& Fischer, 1972; Meier, Cormier \& Quinto-Pozos, 2002; Sandler \& Lillo-Martin, 2006; Johnston \& Schembri, 2007; Brentari, 2010; Zeshan \& de Vos, 2012). However, these questions are often driven by the ontological reductionism of the 'language versus gesture' debate, with the relatively narrow aim of differentiating communicative phenomena that is 'linguistic' from that which is not (see Kendon, 2014 for an overview). Building on the work of Kendon and others, our approach is rather to seek an understanding of the grammar of human multimodality: how do deaf signers and hearing speakers use their available

\footnotetext{
${ }^{1}$ Acknowledgements: Documentation and early development of the Auslan and Australian English archive and corpus was supported by an Australian Research Council grant DP140102124 to Trevor Johnston, Adam Schembri, Kearsy Cormier and Onno Crasborn. Additional corpus enrichment was supported by an ARC Centre of Excellence for the Dynamics of Language grant to Gabrielle Hodge. We are grateful to the Auslan signers and Australian English speakers who contributed to the archive, and the research assistants who worked with us to create it: Stephanie Linder and Sally Bowman. Any errors are our own.
} 
Hodge, G., Sekine, K., Schembri, A. \& T. Johnston. (accepted). Comparing signers and speakers: Building a directly comparable corpus of Auslan and Australian English. Corpora.

communicative heuristics, and how can the resulting semiotic conventions of these communicative ecologies be compared empirically?

The empirical comparison of SL and SpL use is best achieved by directly comparing multimodal composite utterances (Enfield, 2009), and by considering the physical experience of deafness as a factor directly shaping the semiotics available to deaf signers (see Johnston, 1996). However, the strength of the 'all languages are equally complex' paradigm, which encourages an exclusive focus on (often axiomatic) morphosyntactic aspects of language structure, has meant very few studies have used this approach. The most common technique is for multimodal SL data to be compared to unimodal SpL data - which is not a true comparison at all. Yet holistic comparison of the multimodal communication of signers and speakers presents evidence of significant interest. For example, one recent investigation of pronominal pointing in the BSL Corpus and the Travis Smiley American English dataset found that the pointing actions produced by deaf native signers of BSL are more conventionalised and reduced compared to those produced by hearing non-signing speakers of American English (Fenlon, Keane, Cooperrider, Brentari \& Goldin-Meadow, 2016).

The potential for linguists to directly compare SL and SpLs is now better than ever. In the past fifteen years, several SL corpora have been established to document and investigate SLs using corpus methods (Johnston \& Schembri, 2013) ${ }^{2}$. However, the primary intention of most SL corpora has been to address the urgencies of endangered language documentation and corpus-based language description, with an almost exclusive focus on describing the SLs used by native or near-native deaf signers. No corpora have been developed specifically with the aim of directly comparing any specific SL with its ambient SpL.

Researchers are increasingly attending to analyses of SpL used in face-to-face interactions. We have recently seen the development of multimodal SpL corpora (e.g. Carter \& Adolphs, 2008), the expansion of existing theoretical frameworks to accommodate multimodal languaging (e.g. Steen \& Turner, 2013), and wide-ranging studies of language in action (e.g. Haddington, Mondada \& Nevile, 2013). For example, the UCLA NewsScape Archive developed by UCLA and the Distributed Little Red Hen Lab contains more than 250,000 hours of international broadcast digital television and video news programs in American English and other languages, indexed by three billion words of closed captioning, transcripts, and on-screen text ${ }^{3}$.

However, most existing SpL corpora are unimodal, representing only the spoken or written conventions of a given language community (see Xiao, 2008 for an overview). Efforts to describe multimodal language use tend to focus on intensive analysis of a few hearing speakers, rather than the documentation and development of a corpus that is in some way representative of the everyday multimodal talk. Consequently, our understanding of patterns

\footnotetext{
${ }^{2}$ This includes, for example, the Auslan Corpus, Corpus NGT (Nederlandse Gebarentaal), BSL (British Sign Language) Corpus, DGS-Korpus (German Sign Language), Corpus Vlaamse Gebarentaal (Flemish Sign Language), Svenska Teckenspråket (Swedish Sign Language) corpus, the Corpus Project in Colloquial Japanese Sign Language, the Langue des Signes de Belgique Francophone Corpus, and the Corpus de Reference de la Langues des Signes Malienne. See Konrad, 2012 for an overview of SL corpora worldwide, although additional corpus projects have commenced since its publication.

${ }^{3}$ Note the NewsScape Archive consists primarily of television broadcasts, so it is not strictly representative of face-to-face interaction, and access is currently restricted to the UCLA campus community and Red Hen Lab researchers for copyright reasons.
} 
of language use across the broader communicative ecologies of deaf signers and their wider community remains limited, not yet informed by empirical analyses of SL and SpL corpora of each. To redress this imbalance, we undertook the development of the Auslan and Australian English archive and corpus: a digital, audio-visual and machine-readable corpus of face-to-face, multimodal language use produced by deaf native signers of Auslan and hearing native speakers of Australian English.

\section{Characteristics of a directly comparable corpus of multimodal communication}

To resolve these issues, we need a bilingual, multimodal and directly comparable corpus of a deaf SL and its ambient SpL: one that is representative of the repertoire of semiotic resources available to both deaf Auslan signers and hearing Australian English speakers during face-toface communication. The aim is to facilitate the direct comparison of a deaf SL and its ambient SpL by documenting each language ecology using identical sampling frames. Corpus methods are ideal for exploring the patterns and variations of language use, particularly for differentiating patterns of language use common to the whole community from those that are idiosyncratic (Gries, 2009; McEnery \& Hardie, 2012). This is particularly important for investigations of SLs, which are characterised as extremely heterogeneous due to historically lower numbers of native signers and higher numbers of non-native signers (Johnston, 2004).

However, most currently available SL corpora fulfil only one or two of these necessary criteria. All are multimodal in the sense they contain digital recordings of signers from at least waist-up (as opposed to the unimodal audio files documented in many SpL corpora). A small number may be described as bilingual, developed primarily for investigations of first or second language acquisition. These corpora typically contain very small numbers of signers, which restricts any interpretations of language representativeness from analyses. For example, the Child Hong Kong Sign Language-Cantonese Bilingual Corpus represents only two deaf children (Fung, Lam, Mak \& Tang, 2008). Crucially, there are no bilingual, multimodal corpora containing SL built using an identical sampling frame for all languages.

It is the sampling frame of given corpora which facilitates their comparability (McEnery \& Hardie, 2012: 19). A general principle is that the greater the similarity of sampling frame, the greater the comparability potential of the multilingual corpus. All other things being equal (e.g. the demographic of individuals represented in each corpus), a corpus containing data from two languages documented using an identical sampling frame (e.g. where all language consultants are documented in the same way doing the same activities) may therefore be characterised as directly comparable. As existing datasets for comparing multimodal SL and SpLs employ different sampling frames, they have not yet achieved direct comparability. For example, the dataset used by Fenlon et al. (2016) described above draws from two different sampling frames: a video corpus of a SL representing native and near-native signers from different regions in the UK telling stories, being interviewed and conversing with one another, and a corpus of digital broadcasts representing hearing speakers (often celebrities) who were interviewed on television in front of a live audience in the US.

Other bilingual multimodal corpora, such as the spoken German/Swiss German Sign Language corpus of train announcements, are described as parallel rather than comparable (Ebling, 2016). Parallel corpora contain native language L1 source texts and their L2 translations, and cross-language comparison is typically unidirectional (McEnery \& Hardie, 2012: 19). In cross-modal cases, a unimodal spoken or written source text is typically translated into a multimodal signed target text. This is known as a "transmodal" translation and it represents an emerging practice in deaf communities (Leneham, 2005). The potential for multimodal source texts to be transformed into multimodal target texts is also high, but further empirical description of different types of signs (in a neo-Peircian sense) and 
Hodge, G., Sekine, K., Schembri, A. \& T. Johnston. (accepted). Comparing signers and speakers: Building a directly comparable corpus of Auslan and Australian English. Corpora.

processes of semiosis, such as by enriching the corpus described here, are necessary before this can be achieved effectively. The components of existing bilingual multimodal SL corpora are also contingent on some earlier component, rather than intentionally documented at the same time. Our aim in developing the Auslan and Australian English archive and corpus was therefore to fulfil all three criteria (bilingual, multimodal, direct comparability) from the outset.

\section{Documenting deaf signers and hearing speakers for the corpus}

Five pairs of deaf Auslan signers and five pairs of SL-naïve hearing Australian English speakers were filmed undertaking five different activities. All are native or near-native (i.e., first language acquired before age 10) language users who have been living in Melbourne for at least ten years. Individual consultants in each pair are social intimates (siblings, good friends, or colleagues), and all pairs already knew the research assistant who directed their session. Deaf native signer Stephanie Linder facilitated the pairs of deaf signers, with technical support from the first author (also deaf). Hearing native speaker Sally Bowman facilitated the pairs of hearing speakers, with technical support from the second author (also hearing). Table 1 details how language consultants were roughly balanced for age and gender, while also representing a degree of community heterogeneity within the limits of the small sample size (see $\$ 4.2$ regarding the limitations of the corpus). Consultants are also balanced in terms of educational background, as nine deaf signers and eight hearing speakers hold tertiary qualifications such as a Graduate Diploma or Bachelor of Arts.

\section{[TABLE 1 HERE]}

Pairs of language consultants were guided to complete the following activities: (a) discussion of twelve social dilemmas (including eight adapted from Zwets, 2014); (b) the Family Problems task (San Roque et al, 2012); (c) retelling the written Aesop's Fable The Boy Who Cried Wolf and the illustrated book Frog, Where Are You? (Mayer, 1969) ${ }^{4}$; (d) free conversation with snacks and refreshment; (e) an elicitation task using eight silent movie vignettes (adapted from So et al, 2005) ${ }^{5}$; and (f) the Stacks and Squares task (Cooperrider et al, 2014).

\section{Building the Auslan and Australian English Archive and Corpus}

\subsection{Filming technicalities and file name conventions}

All activities were filmed with two Canon FS11E digital camcorders and one GoPro Hero3 positioned above language consultants from a boom, capturing a bird's-eye view of the proceedings (Figure 1). One Sony TCM-500DEV multidimensional audio recorder was connected to Camera B to capture superior audio recordings.

\section{[FIGURE 1 HERE]}

Filming activities with the ten pairs resulted in approximately fifteen hours of audio-visual recordings, i.e. an average of 90 minutes of recordings for each pair. All audio-visual data was synced and edited using Adobe Premier Pro CC 2015 and Adobe Media Encoder CC 2015 software. The synced recordings were then edited per the activities summarised in Table 2 , which resulted in approximately 1,000 clips from three cameras. All edited clips were

\footnotetext{
${ }^{4}$ Only hearing speakers completed the Frog and Wolf retelling tasks, as Auslan retellings of these narratives were previously archived in the Auslan Corpus and have since been heavily enriched with annotations (see e.g. Ferrara \& Johnston, 2014).

${ }^{5}$ Each hearing speaker retold four vignettes with speech and four without speech.
} 
exported as mpeg2 (semi-archival albeit lossy quality) and H.264 codec in mp4 container (enabling reasonable ELAN functionality with either Mac or PC).

\section{[TABLE 2 HERE]}

All mpeg2 and mp4 clips are archived at The Language Archive (TLA) at the Max Planck Institute for Psycholinguistics. The Auslan Family Problems mpeg2 and mp4 clips (the ASF $12 \mathrm{a}-\mathrm{d}$ files) have also been archived at PARADISEC as part of the Social Cognition Parallax Interview Corpus (SCOPIC) project at the ARC Centre of Excellence for the Dynamics of Language. All archived data is available to registered academic users of each archive. Each file in the archive is uniquely identified according to the conventions outlined in Table 3 . To facilitate the future integration of the new Auslan data into the existing Auslan Archive deposited at ELAR, all activities were intentionally numbered to follow on from the activities listed in the Auslan Corpus (see Johnston, 2016).

\section{[TABLE 3 HERE]}

\subsection{Limitations of the Auslan and Australian English archive}

Despite our exacting standards for building a bilingual, multimodal and directly comparable corpus, the resulting archive is constrained by two major limitations. Firstly, technical requirements meant it was necessary to adhere to the traditional 'language lab' model of language documentation: a private room facilitating the high-quality recording of all aspects of multimodal communication. Findings resulting from corpus analyses should therefore be supplemented with more ethnographical understandings of deaf signers and hearing speakers, especially with respect to their geographical and social mobility (see, for example, Kusters, 2010). We also experienced some technical difficulties with the use of the GoPro overhead camera: its sensitivity made it somewhat unreliable during filming, and a small number of activities are consequently missing an overhead camera feed.

Secondly, limited time and money meant the inclusion of only ten deaf signers and ten hearing speakers in the archive, a small number compared to existing SL and SpL corpora. It may be difficult to differentiate degrees of conventionality from extreme idiosyncrasy of multimodal languaging across these small numbers. However, we suggest this limited representation may be mitigated by mapping analyses with those from other, larger corpora such as the Auslan Corpus and the Australian National Corpus (see §4.3).

\subsection{Development pathway into a directly comparable corpus of SL and SpL}

There are two paths by which the Auslan and Australian English archive can develop into a directly comparable corpus. Firstly, by applying the annotation conventions developed for the Auslan Corpus to both the Auslan and Australian English multimodal data (Johnston, 2016). This method has been trialled with an early analysis of the composite utterances produced by the hearing speakers in the archive as they retell the Frog and Wolf narratives. In total, ten speakers produced 115 tokens of manual co-speech gestures depicting movement, shape and/or concepts during 20.25 minutes of data - about one token every eleven seconds. Compare with the 1,031 partly-lexicalised depicting signs produced by forty deaf signers during 83.6 minutes of Auslan Frog and Wolf retellings - about one token every five seconds. The following examples compare Auslan and Australian English composite utterances during which the language consultant locates and depicts the glass jar in Frog: 
Hodge, G., Sekine, K., Schembri, A. \& T. Johnston. (accepted). Comparing signers and speakers: Building a directly comparable corpus of Auslan and Australian English. Corpora.

[FIGURE 2 HERE]

$\begin{array}{lll}\text { GLASS DEPICTING SIGN: SPHERICAL-OBJECT } & \text { FROG IN } & \text { PT:LOC } \\ \text { "A glass bowl there, } & \text { a frog inside } & \text { there." } \\ \text { (Auslan Corpus SLRBc7a_CLU\#002) } & & \end{array}$

[FIGURE 3 HERE]

Secondly, by supplementing analyses resulting from these enrichments with analyses of data from the much larger Auslan Corpus, and even unimodal Australian English corpora (e.g. ICE-AUS, Australian National Corpus, AusTalk Corpus). Figure 4 depicts this potential for analyses from larger corpora to be mapped to findings from our much smaller corpus, thus alleviating issues with sample size and representativeness. Hypotheses developed from analysis of one set of corpus data could be investigated on a micro/macro scale with respect to multi/unimodality, whichever the case may be. For example, is $X$ a common speech construction in spoken Australian English or is it simply one person's unique phrasing, and does this affect our understanding of their multimodal utterance composition?

\section{[FIGURE 4 HERE]}

\section{Research significance}

In seeking an understanding of the grammar of human multimodality, we must be able to describe the semiotic conventions of SL and SpL communicative ecologies empirically. The starting point for this description is to directly compare the composite utterances of signers and speakers. The documentation of the bilingual, multimodal and directly comparable Auslan and Australian English archive and its development into a machine-readable corpus enables this comparison on an unprecedented scale. In concluding this paper, we can now return to the question which led to its creation: how do deaf signers and hearing speakers use their available communicative heuristics, and what are the semiotic conventions of these ecologies? From this we can ask many other questions that have mostly eluded direct comparability on a large scale: are the symbolic indexicals (such as pointing actions) produced by signers and speakers alike in form and (pluri)functionality? Are strategies of depiction and enactment patterned in the same way in composite utterances, regardless of the presence or absence of speech? If so, do they differ in degrees of conventionality and entrenchment, and what does this say about the nature of human languages and linguistic diversity?

The activities documented in the archive provide fertile ground for further investigation of these questions. For example, the Australian English Frog and Wolf retellings and conversation data may be readily compared with existing analyses of composite utterances in the Auslan Corpus. The conversation and Family Problems Task data facilitates comparison of multimodal talk of signers and speakers while doing other things, such as eating and drinking, or manipulating picture cards. The Family Problems and Stacks \& Squares data enables typological comparison of both Auslan and Australian English with other languages that have been documented using this task (in fact there are over twenty diverse languages in the SCOPIC project alone, see Barth \& Evans, 2017). The social dilemma data are well suited to both comparison of different blending strategies for talking about people, things and events occurring in specific geographic locations, and the silent movie vignette data enables comparison of deaf signers with hearing non-signers communicating with or without speech. Given time and corpus enrichment, questions of how patterns of multimodal SL use compare to multimodal $\mathrm{SpL}$ use may finally be answered. 


\section{Tables and Figures}

Table 1. Language consultants represented in the Auslan and Australian English archive

\begin{tabular}{lllll}
\hline Age range & $20-30$ & $31-45$ & $46-65$ & Total \\
\hline Deaf female & 0 & 4 & 1 & 5 \\
Deaf male & 0 & 3 & 2 & 5 \\
Hearing female & 2 & 1 & 2 & 5 \\
Hearing male & 2 & 2 & 1 & 5 \\
\hline Total & 4 & 10 & 6 & 20 \\
\hline
\end{tabular}

Table 2. Documented tasks in the Auslan and Australian English archive

\begin{tabular}{lll}
\hline Activity & Activity number & Activity code \\
\hline Social dilemma 1-12 & 11 & $\mathrm{c} 11 \mathrm{a}-\mathrm{c} 111$ \\
Family Problems $1-3 \mathrm{~b}$ & 12 & $\mathrm{c} 12 \mathrm{a}-\mathrm{c} 12 \mathrm{~d}$ \\
Retelling Wolf & 2 & $\mathrm{c} 2 \mathrm{a}$ \\
Free conversation & 5 & $\mathrm{c} 5$ \\
Retelling Frog & 7 & $\mathrm{c} 7 \mathrm{a}$ \\
Vignette $1-8$ & 13 & $\mathrm{c} 13 \mathrm{a}-\mathrm{c} 13 \mathrm{~h}$ \\
Stacks \& Squares $0-8$ & 14 & $\mathrm{c} 14 \mathrm{practice}-\mathrm{c} 14 \mathrm{~h}$ \\
\hline
\end{tabular}

Table 3. File naming conventions for the Auslan and Australian English archive

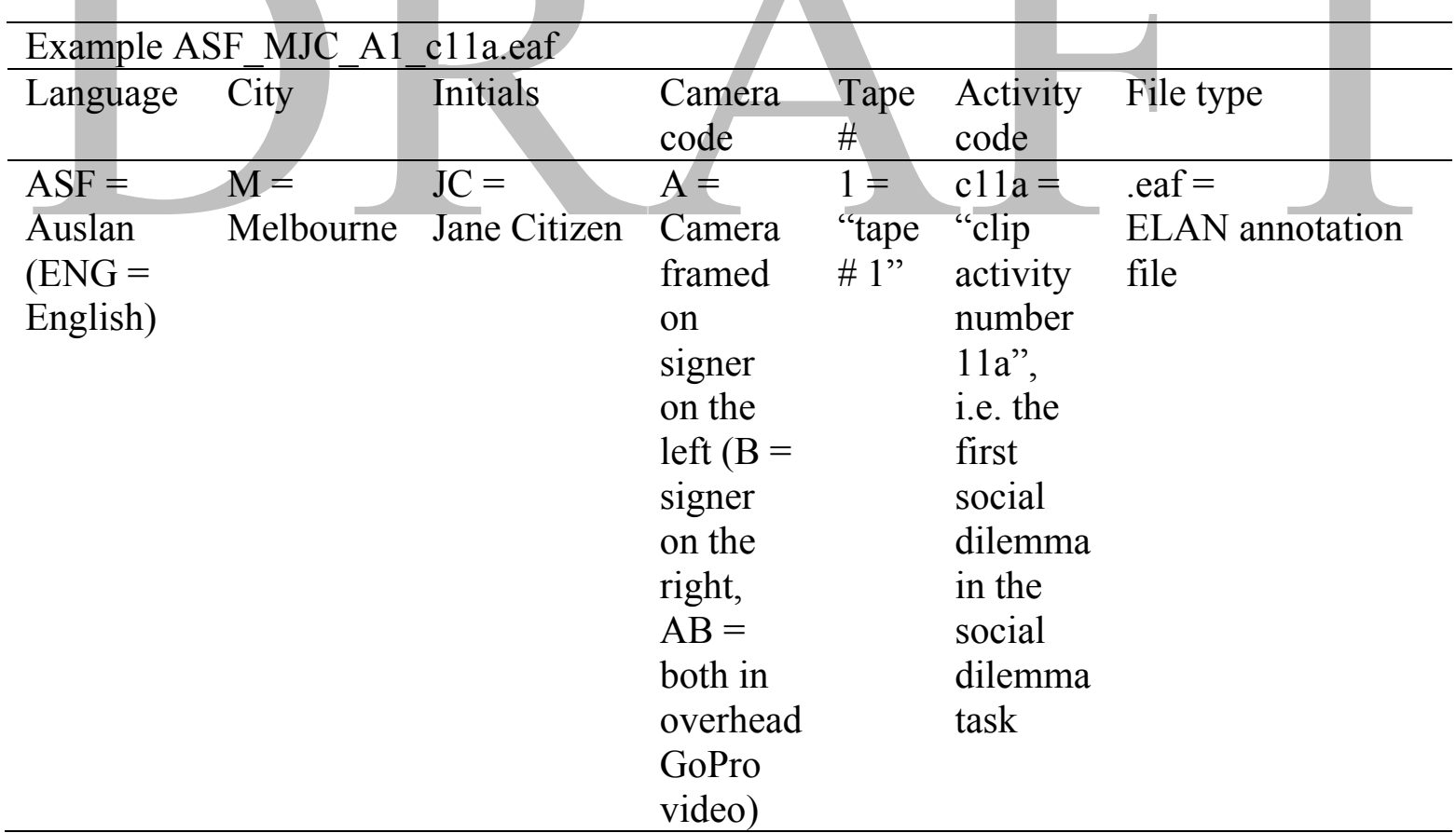


Hodge, G., Sekine, K., Schembri, A. \& T. Johnston. (accepted). Comparing signers and speakers: Building a directly comparable corpus of Auslan and Australian English. Corpora.

Figure 1. View of language consultants filmed with Camera A, AB and B
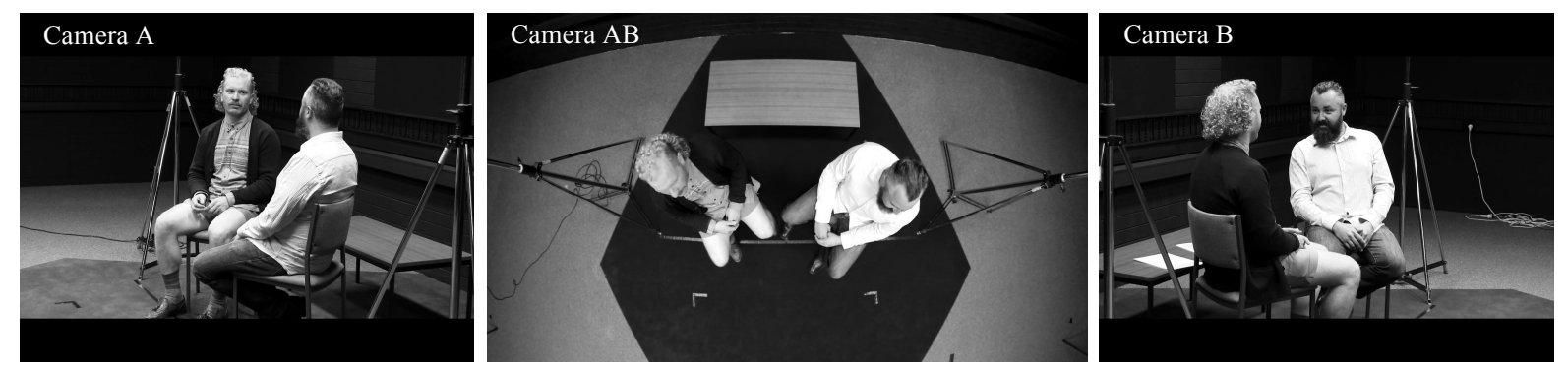

Figure 2. Example of an Australian English composite utterance in which the consultant locates and depicts the glass jar in Frog, Where Are You?

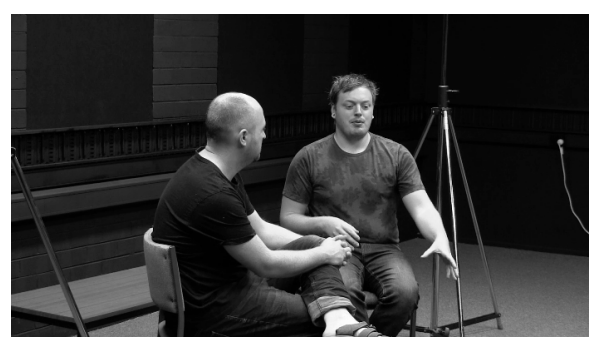

Figure 3. Example of an Auslan composite utterance in which the consultant locates and depicts the glass jar in Frog, Where Are You?

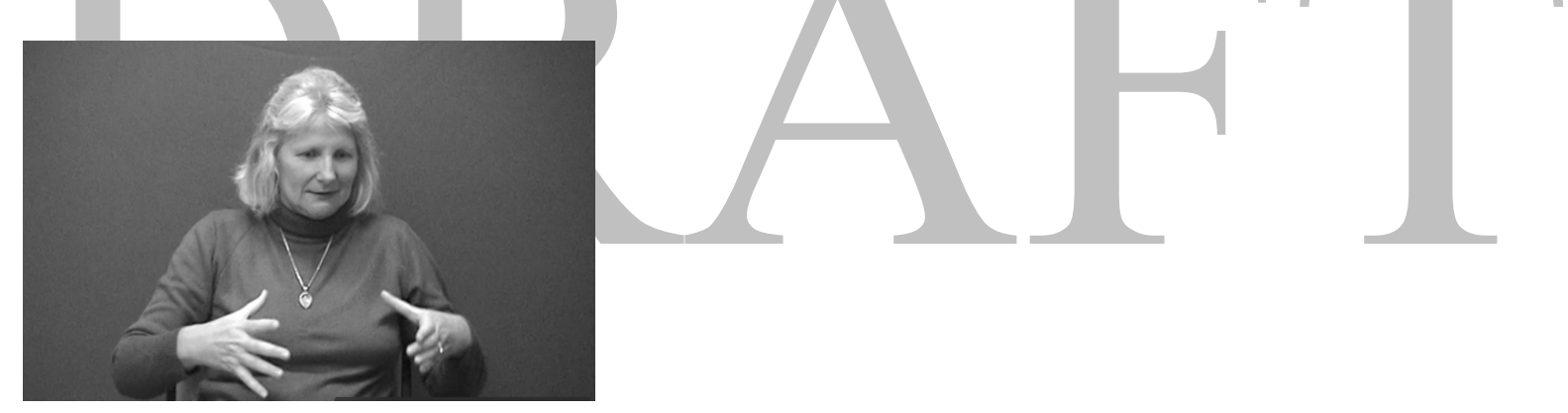

Figure 4. Potential for cross-corpora feeds (solid line = multimodal corpora; dashed line = unimodal corpora)

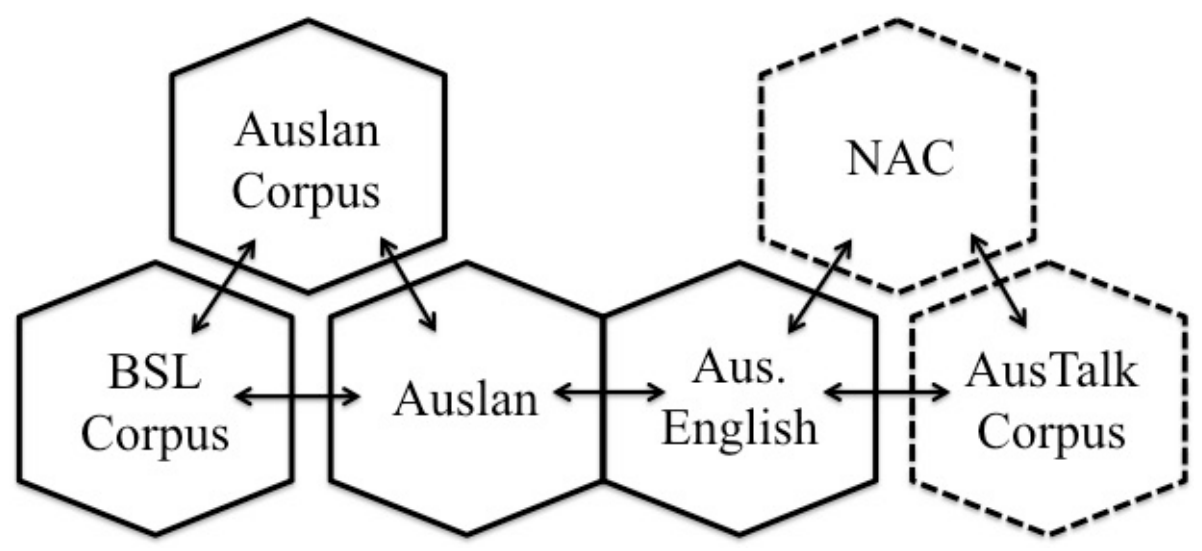




\section{References}

Barth, D. \& N. Evans. 2017. 'SCOPIC Design and Overview'. Language Documentation \& Conservation Special Publication No. 12: Social Cognition Parallax Interview Corpus (SCOPIC), pp. 1-21. http://hdl.handle.net/10125/24742

Bellugi, U. \& S. Fischer. 1972. 'A comparison of signed language and spoken language'. Cognition (1): 173-198.

Brentari, D. 2010. Sign Languages. Cambridge: Cambridge University Press.

Carter, R. \& Adolphs, S. 2008. 'Linking the verbal and visual: New directions for Corpus Linguistics'. Language and Computers, 64: 275-291.

Cooperrider, K., Nunez, R. \& J. Slotta. 2014. 'The protean pointing gesture: Variation in a building block of human communication' in P. Bello, M. Guarini, M. McShane, \& B. Scassellati (eds.), Proceedings of the 36th Annual Meeting of the Cognitive Science Society, pp. 355-360. Austin, TX: Cognitive Science Society.

Ebling, S. 2016. 'Building a parallel corpus of German/Swiss German Sign Language train announcements'. International Journal of Corpus Linguistics, 21(1): 116 - 129. DOI 10.1075/ijcl.21.1.06ebl

Enfield, N. 2009. The Anatomy of Meaning: Speech, Gesture, and Composite Utterances. Cambridge: Cambridge University Press.

Fenlon, J., J. Keane, K. Cooperrider, D. Brentari, \& S. Goldin-Meadow. 2016. 'Comparing pronominal signs with pointing gestures'. Presented at the International Society for Gesture Studies conference, 18 - 22 July. Paris, France.

Ferrara, L. \& T. Johnston. 2014. 'Elaborating who's what: a study of constructed action and clause structure in Auslan Australian sign language'. Australian Journal of Linguistics 34(2): 193-215.

Fung, C., Lam, S., Mak, J., Tang, G. 2008. 'Establishment of a corpus of Hong Kong Sign Language acquisition data: From ELAN to CLAN'. In Crasborn, O., E. Efthimiou, T. Hanke, E. D. Thoutenhoofd \& I. Zwitserlood (eds.). LREC 2008 Workshop Proceedings. W 25: $3^{\text {rd }}$ Workshop on the Representation and Processing of Sign Languages: Construction and Exploitation of Sign Language Corpora. (pp. 17-21). Paris: ELRA. [Online resource; URL: http://www.lrecconf.org/proceedings/lrec2008/workshops/W25_Proceedings.pdf]

Gries, S. T. 2009. What is corpus linguistics? Language and Linguistics Compass, 3(5): 1225-1241.

Haddington, P., L, Mondada \& M. Nevile (eds.). 2013. Interaction and mobility: Language and the body in motion. Berlin: Walter de Gruyter.

Johnston, T. 1996. Function and medium in the forms of linguistic expression found in a sign language. In W. H. Edmondson \& R. B. Wilbur (eds.), International Review of Sign Linguistics (Vol. 1, pp. 57-94). Mahwah, NJ: Lawrence Erlbaum.

Johnston, T. 2004. 'W(h)ither the deaf community? Population, genetics and the future of Australian Sign Language'. Sign Language Studies, 6(2): 137-173.

Johnston, T. 2016. Auslan Corpus Annotation Guidelines [November 2016 version]. Macquarie University, Sydney. Available at: http://www.auslan.org.au/about/annotations/

Johnston, T. \& A. Schembri. 2007. Australian Sign Language (Auslan): An introduction to sign language linguistics. Cambridge: Cambridge University Press.

Johnston, T., \& A. Schembri. 2013. 'Corpus analysis of sign languages' in C. A. Chapelle (Ed.). The Encyclopaedia of Applied Linguistics. UK: Blackwell Publishing Ltd. DOI: 10.1002/9781405198431.wbeal0252

Kendon, A. 2014. Semiotic diversity in utterance production and the concept of 'language'. Phil. Trans. R. Soc. B, 369: 20130293. DOI: 10.1098/rstb.2013.0293 
Hodge, G., Sekine, K., Schembri, A. \& T. Johnston. (accepted). Comparing signers and speakers: Building a directly comparable corpus of Auslan and Australian English. Corpora.

Konrad, R. 2012. Sign language corpora survey [June 2012 version]. Retrieved 15-12-16 from http://www.sign-lang.uni-hamburg.de/dgs-korpus/index.php/sl-corpora.html

Kusters, A. 2010. 'Deaf on the lifeline of Mumbai'. Sign Language Studies, 10(1): 36-68.

Leneham. M. (2005). The Sign Language interpreter as translator: Challenging traditional definitions of translation and interpreting. Deaf Worlds, 21(1): 79-101.

Mayer, M. 1969. Frog, where are you? New York: Dial Press.

McEnery, T., \& A. Hardie. 2012. Corpus Linguistics: Method, Theory and Practice. Cambridge: Cambridge University Press.

Meier, R., K. Cormier \& D. Quinto-Pozos. 2002. Modality and structure in signed and spoken languages. Cambridge: Cambridge University Press.

Sandler, W., \& D. Lillo-Martin. 2006. Sign language and linguistic universals. Cambridge: Cambridge University Press.

San Roque, L., L. Gawne, D. Hoenigman, J. Miller, A. Rumsey, S. Spronck, A. Carroll \& N. Evans. 2012. 'Getting the Story Straight: Language Fieldwork Using a Narrative Problem-Solving Task'. Language Documentation \& Conservation 6: 135-174. http://hdl.handle.net/10125/4504

So, W. C., M. Coppola, V. Licciardello \& S. Goldin-Meadow. 2005. 'The seeds of spatial grammar in the manual modality'. Cognitive Science 29: 1029-1043.

Steen, M. \& M. Turner. 2013. 'Multimodal Construction Grammar' in M. Borkent, B. Dancygier \& J. Hinnell (eds.). Language and the Creative Mind, pp. 255-274. Stanford, CA: CSLI Publications.

Xiao, R. Z. 2008. 'Well-known and influential corpora' in A. Lüdeling \& M. Kytö (Eds.). Corpus Linguistics: An International Handbook. Handbooks of Linguistics and Communication Science, 1. Berlin: Mouton de Gruyter.

Zeshan, U. \& C. de Vos. 2012. Sign languages in village communities: Anthropological and linguistic insights. Sign Language Typology Series No. 4. Berlin: De Gruyter Mouton \& Nijmegen: Ishara Press.

Zwets, M. 2014. Locating the difference: A comparison between Dutch pointing gestures and pointing signs in Sign Language of the Netherlands. Utrecht: LOT. 